\title{
Reconstruction of Ares I-X Integrated Vehicle Rollout Loads
}

\author{
Dr. Matthew K. Chamberlain ${ }^{1}$ and Dr. Steven R. Hahn ${ }^{2}$ \\ Dynamic Concepts, Inc., Huntsville, AL 35806, USA
}

\begin{abstract}
The measurements taken during the Ares I-X test flight provided a unique opportunity to assess the accuracy of the models and methods used to analyze the loads and accelerations present in the planned Ares I vehicle. During the rollout of the integrated vehicle from the Vehicle Assembly Building (VAB) to the launch pad, the vehicle and its supporting structure are subjected to wind loads and the vibrations produced by the crawler-transporter (CT) that is carrying it. While the loads induced on the vehicle during this period are generally low relative to those experienced in flight, the rollout is a period of operation of primary interest to those designing both the ground support equipment and the interfaces between the launch vehicle and its supporting structure. In this paper, the methods used for reconstructing the loads during the rollout phase are described. The results generated are compared to measured values, leading to insight into the accuracy of the Ares I assessment techniques.
\end{abstract}

\section{Notice to readers:}

The predicted performance and certain other features and characteristics of the Ares I and Ares I-X launch vehicles are defined by the U.S. Government to be Sensitive But Unclassified (SBU). Therefore, details have been removed from selected plots and figures

\section{Nomenclature}

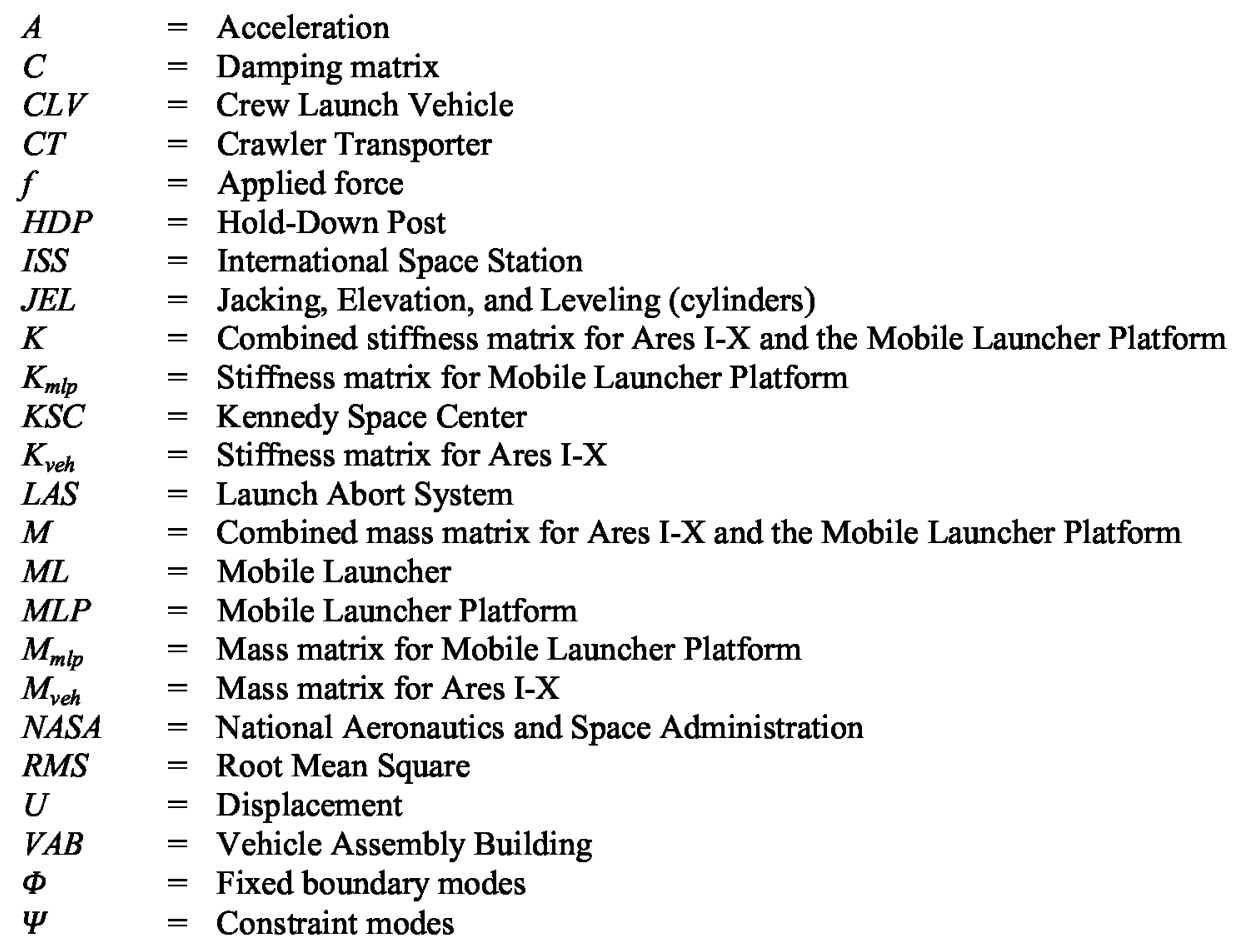

\footnotetext{
${ }^{1}$ Engineer / Scientist, 8700 Odyssey Dr, Suite 202, Huntsville, AL 35806, AIAA Member

${ }^{2}$ Senior Engineer / Scientist, 8700 Odyssey Dr, Suite 202, Huntsville, AL 35806, AIAA Member
} 


$$
\begin{array}{ll}
\Psi_{C B} & =\text { Craig-Bampton Transformation Matrix } \\
\eta & =\text { Modal displacement } \\
\gamma & =\text { Tukey power factor }
\end{array}
$$

\section{Introduction \& Background}

$\mathrm{T}$ he Ares I-X developmental test flight on October 28, 2009 was meant as a demonstration of the characteristics and handling of the future Ares I crew launch vehicle (CLV). As a part of the Constellation program under development by of the National Aeronautics and Space Administration's (NASA), the two-stage Ares I booster would be used to place the Orion spacecraft in orbit for missions to the International Space Station (ISS) as well as the lunar surface. The Ares I-X vehicle was heavily instrumented during and prior to its flight, providing invaluable data to the teams involved in the ongoing design-analysis cycle for Ares I. This paper describes how data collected prior to the launch of Ares I-X was used in validating and improving the analysis methods used to assess loads during the rollout of future Ares I vehicles.

Prior to launch from launch complex 39 at the Kennedy Space Center (KSC) in Florida, launch vehicles must be transported from the Vehicle Assembly Building (VAB) to either of the two launch pads more than three miles away by a crawler-transporter (CT) vehicle as shown in Fig. 1 . The CT picks up both the vehicle and

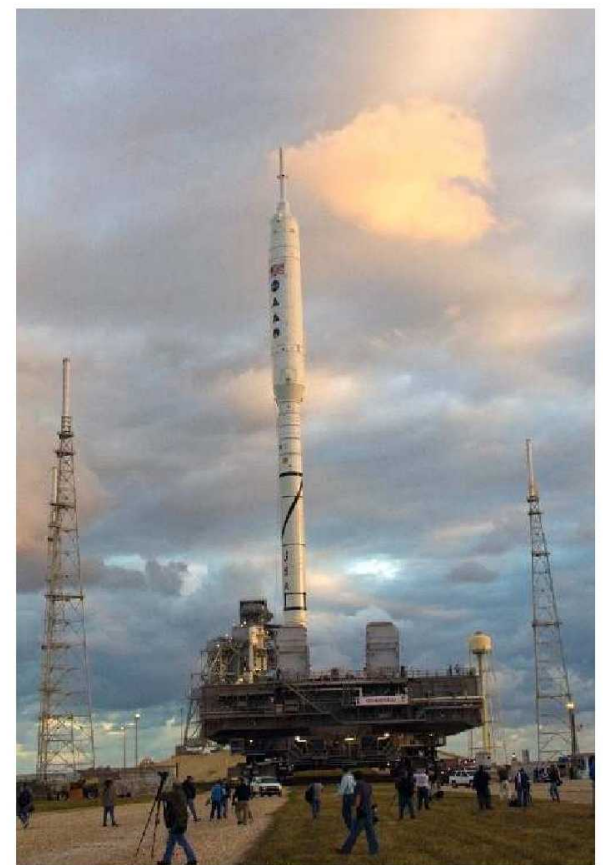

Figure 1. Ares I-X at End of Rollout. the platform that supports it: either a Multiple Launcher Platform (MLP) in the case of the Space Shuttle and Ares I$\mathrm{X}$ or a Multiple Launcher (ML) in the case of Ares I. The as-yet incomplete ML differs from the MLP in that it includes a large tower and two stabilizing arms that would restrain an Ares I vehicle from the point at which it is stacked up until launch.

The phase of operation from the point at which the CT lifts the vehicle up in the VAB to the point at which it lowers them at the launch pad is the focus of this paper. The launch vehicle itself is not expected to experience peak loads or moments during the rollout phase of operations, however prior analysis has suggested that attention be paid to the points at which the launch vehicle and the ML or MLP are in contact. Of particular concern in the rollout analysis are the following quantities:

- The axial loads on the hold-down posts (HDP) on the ML or MLP which support the weight of the launch vehicle. To ensure stability and the orientation of the vehicle at launch, it is important to ensure that the vehicle never becomes unseated from a HDP.

- The loads present in the aft skirt of the first stage of the vehicle where it is in contact with the HDPs.

- The loads present in the stabilizer arms that connect the Ares I vehicle to the ML tower. These structures were not present for the rollout of Ares I-X.

During the rollout of Ares I-X, over eight hours of acceleration and strain time histories were recorded at key points on the MLP and the vehicle itself. Many of the same methods utilized in a typical Ares I analysis cycle were reused in an attempt to reconstruct the loads measured. The information provided by this exercise has proved valuable in validating the modeling methods, simulation techniques, and conservatism of the typical Ares I rollout analysis procedure. In Section II of this paper, the approach used to model the MLP and Ares I-X during rollout is described. In The procedure used to simulate rollout is explained $\mathrm{n}$ Section III while the results and the implications of those results are discussed in Sections IV and V respectively.

\section{Modeling and Boundary Conditions}

The finite element models of the MLP and Ares I-X used in this reconstruction Craig-Bampton reduced order representations produced by NASA. The Ares I-X model had 536 physical and 966 generalized modes while the MLP had 363 physical and 966 generalized modes. The vehicle was assumed to be pinned to the MLP at the four HDPs. Stiff springs with were applied to all three translational degrees of freedom at the HDPs to serve as a penalty function for violating the constraint that the vehicle sit still on the HDPs. This stiffness value was specified to provide the minimum stiffness necessary to adequately enforce the constraint while avoiding introducing any illconditioning through loss of precision and round-off error. 
The combined MLP and vehicle model stiffness matrices (already reduced using the Craig-Bampton method) were assembled as follows:

$$
\begin{aligned}
M & =\left[\begin{array}{cc}
M_{\text {veh }} & 0 \\
0 & M_{m l}
\end{array}\right] \text { (1) } \\
K & =\left[\begin{array}{cc}
K_{\text {veh }} & 0 \\
0 & K_{m l}
\end{array}\right] \text { (2) }
\end{aligned}
$$

Prior to being picked up by the CT inside the VAB for rollout, the Ares I-X vehicle was stacked on top of an MLP which itself was supported at six points, as shown in Fig. 2. Supports at the same six points were used at the launch pad after rollout. While modeling the effects of gravity before and during crawler pickup (see Section III), the combined model was grounded at these six points using very stiff springs. Prior to rollout, the CT was moved into position below the MLP and raised using four sets of jacking, elevation, and lifting (JEL) cylinders. Thus, as it was carried by the CT, the combined structure of the MLP and the launch vehicle were supported at four points (see Fig. 2). As discussed in Section III, triaxial accelerometers at the top of the JEL cylinders, just below the interface between the CT and the MLP measured base drive accelerations used in the simulation to drive the combined model. Lacking sufficient models of the CT and the behavior of the JEL cylinders, the dynamics of the CT were not considered in this work.
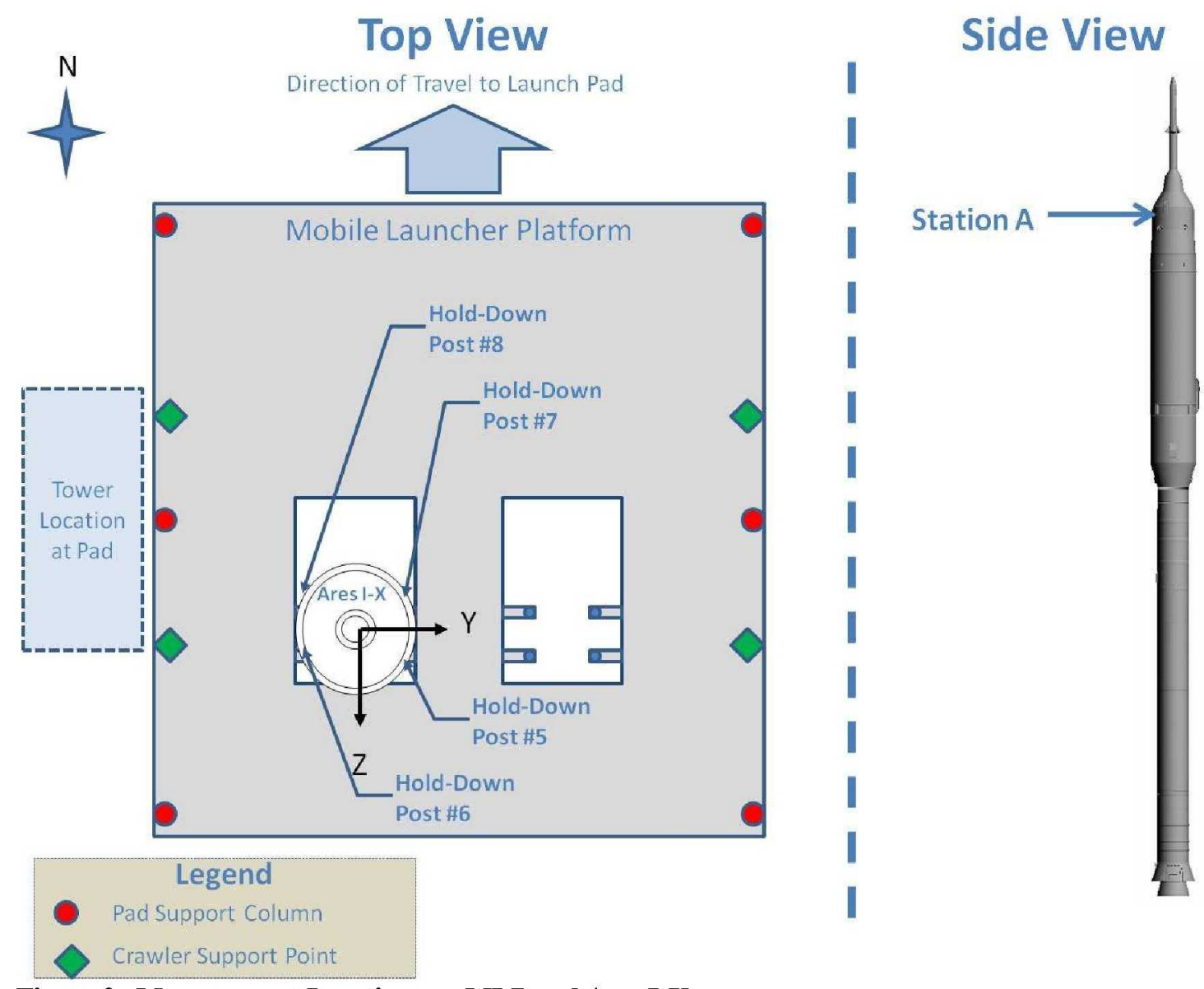

Figure 2. Measurement Locations on MLP and Ares I-X.

\section{Method}

The methods used for modeling and simulating the rollout of Ares I-X are similar to those used to analyze Ares I during a loads cycle but with one key difference. Whereas in a loads cycle, the combined finite element model of the ML and Ares I are subjected to worst-on-worst combinations of gravity, crawler base drive, and wind loading, Ares $\mathrm{I}-\mathrm{X}$ is assumed here to be subject to the measured base drive and gravity alone. During the early phases of rollout, 
winds were low enough that this was thought to be a safe assumption. Also, during a loads cycle, loads transformation matrices are utilized to derive loads throughout the vehicle. With only a few points on the vehicle instrumented during the Ares I-X rollout, only a few values were derived directly from the dynamic responses of the system.

The key steps in the reconstruction of the Ares I-X rollout thus were the processing of measured data, the simulation of the effects of base drive accelerations, correction for overturning moment in the vehicle, and simulation of the effects of gravity and the changing boundary conditions on the MLP. These steps are described in the three subsections that follow.

\section{A. Processing of Measured Data}

Three sets of data are utilized in this reconstruction effort. Accelerations in three directions at each of the four MLP/CT interface points (see Fig. 2 and Fig. 3) are used to simulate the base drive from the crawler. Strain readings on the HDPs and accelerations measured at Station A (see Fig. 2) were used to validate the simulated results.

With over eight hours of rollout data available, a subset of time had to be selected for study. Because initial inspection of strain readings in the hold-down posts suggested high loads when the crawler slowed down to cross roads, it was judged desirable to study a portion of the rollout that included at least one slow-down period and one steady speed period. Previous loads analysis cycles for Ares have shown that when winds are low, their impact on the overall loads on the vehicle and MLP are negligible compared to that of the base drive. This fact made it desirable to study a period of time in which winds were low.

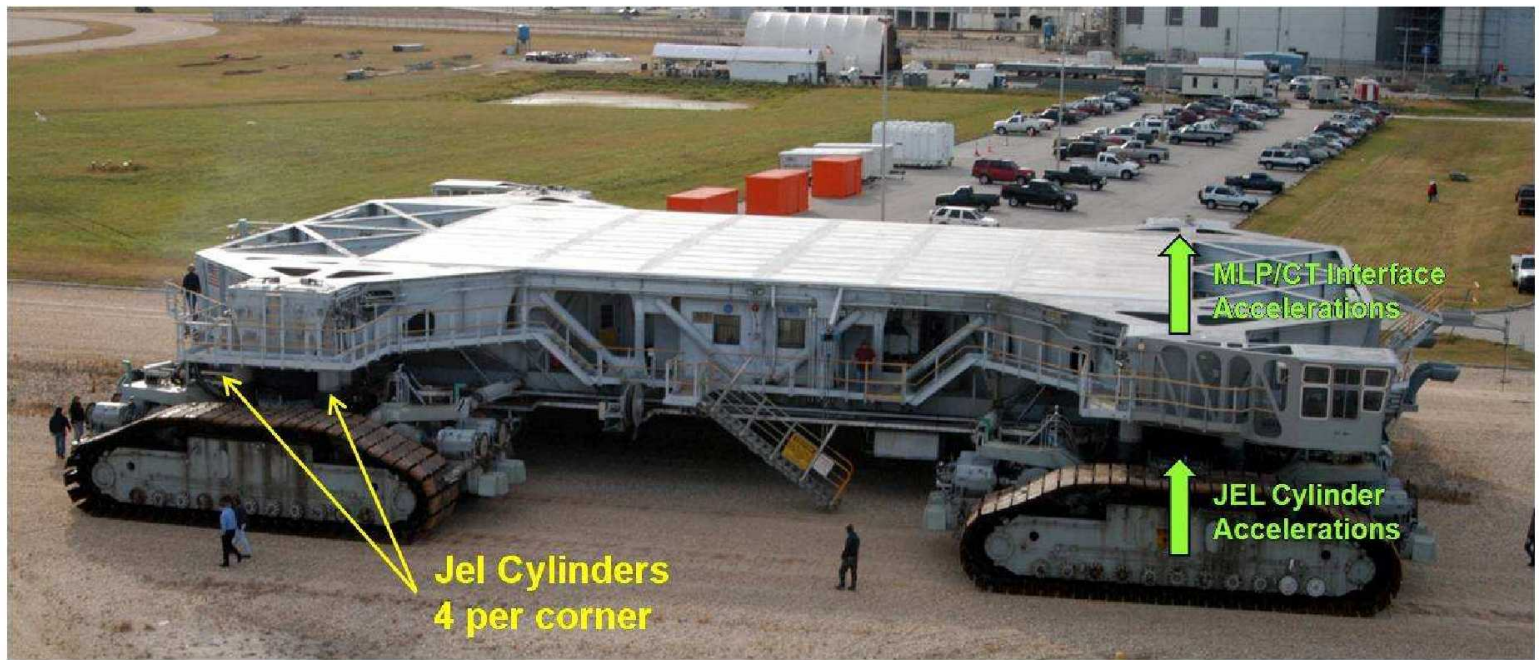

Figure 3. Crawler Transporter Showing JEL Cylinder and MLP/CT Interfaces.

Wind data from the corner of the MLP (measurement and crawler velocity data from GPS readings are plotted in Fig. 4. Inspection of this figure shows that winds were quite low during much of the first half of the rollout of Ares I-X. Winds suddenly began picking up around the $10,000 \mathrm{sec}$ mark. The CT had to slow down twice before this point because two road crossings. For this reason, a time period between $3,500 \mathrm{sec}$ and $10,000 \mathrm{sec}$ was selected for study in this reconstruction effort. This time period represents over 108 minutes of data -slightly more than the length of time studied in previous Ares I loads analysis cycles. 


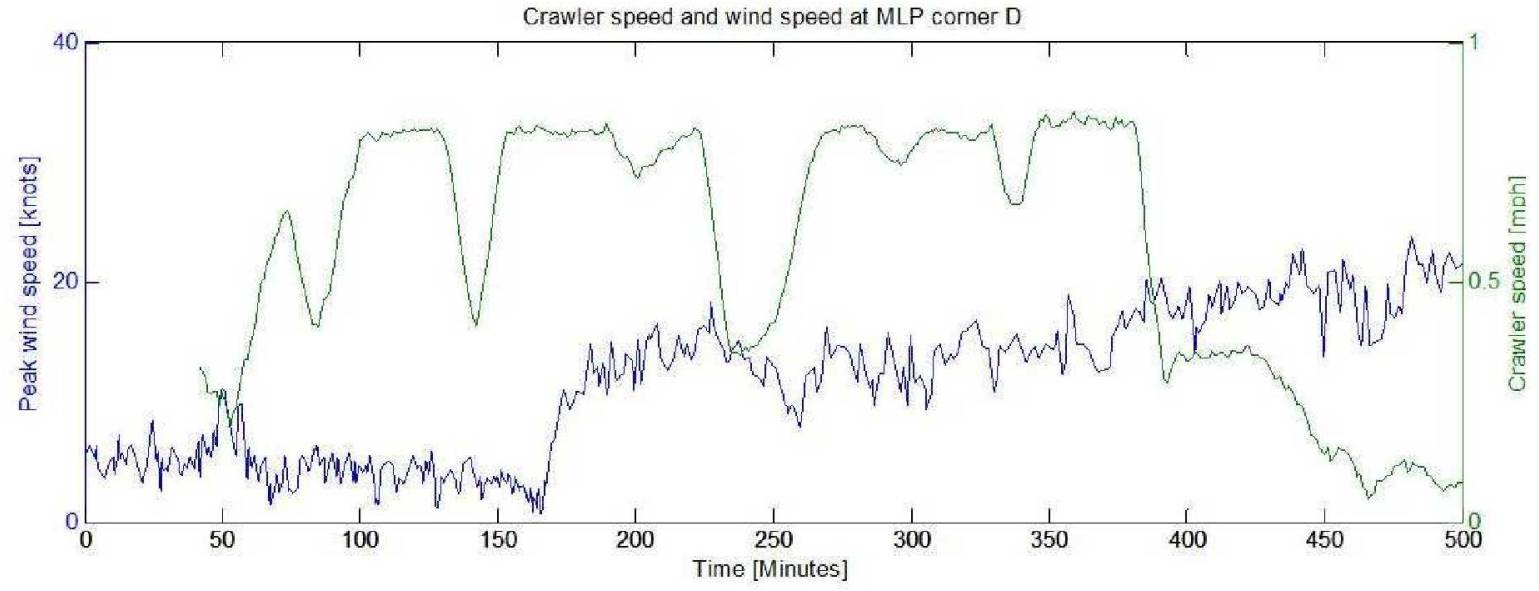

Figure 4. Crawler Velocity and Wind Speed During Rollout.

The accelerations due to the base drive at the MLP/CT interface for the Ares I-X rollout were measured in G's at $100 \mathrm{~Hz}$ using a series of twelve accelerometers numbered KSRDG301A through KSRDG312A. Filtering the base drive accelerations for use in driving the finite element models of the structure presented a challenge since the nominal noise floor of the accelerometers exceeded the first two bending modes of the structure. This fact is demonstrated in a power spectral density (PSD) plot of the Z-axis accelerations at one of the MLP/CT attach points in blue and the extrapolated noise floor for the accelerometer in green in Fig. 5. The interaction of these two lines above the second bending mode throws doubt on the readings for the accelerometer at the values of the first two bending modes. Indeed, the general trend of the PSD at frequencies beyond this point can be seen to run parallel to the extrapolated noise floor. 


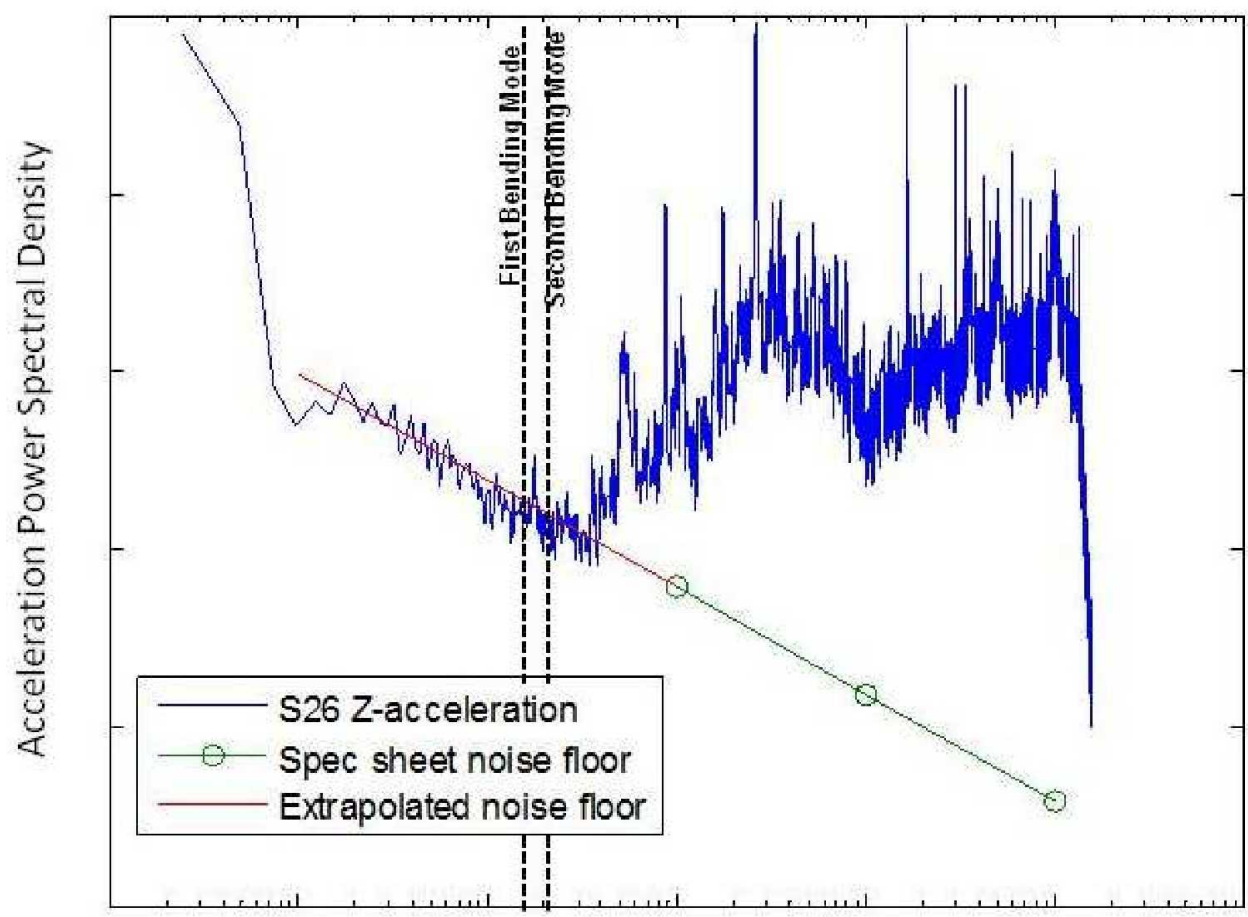

Frequency

Figure 5. Measured MLP/CT Interface Z-Acceleration.

Because of the importance of the first two modes and the doubt surrounding the accelerometer readings around those frequencies, a scaled Tukey Window filtering approach was used on the raw measured data. This approach was meant to allow in some, but not all of the low frequency signals and is computed as follows:

noise, $n \propto f^{-\alpha}$ grows with smaller frequencies

signal, $s \propto f^{\beta}$ decreases with smaller frequencies

$$
\text { scale }=\frac{s}{s+n} \propto \frac{f^{\beta}}{f^{-\alpha}+f^{\beta}}=\frac{\left(f / f_{0}\right)^{\gamma}}{1+\left(f / f_{0}\right)^{\gamma}}
$$

where $\gamma$ is the power that controls how quickly the scaling drops off at low frequencies and fo is the frequency at which the scaling factor is $1 / 2$. An example of the scale factor is plotted in Fig. 6 below. 


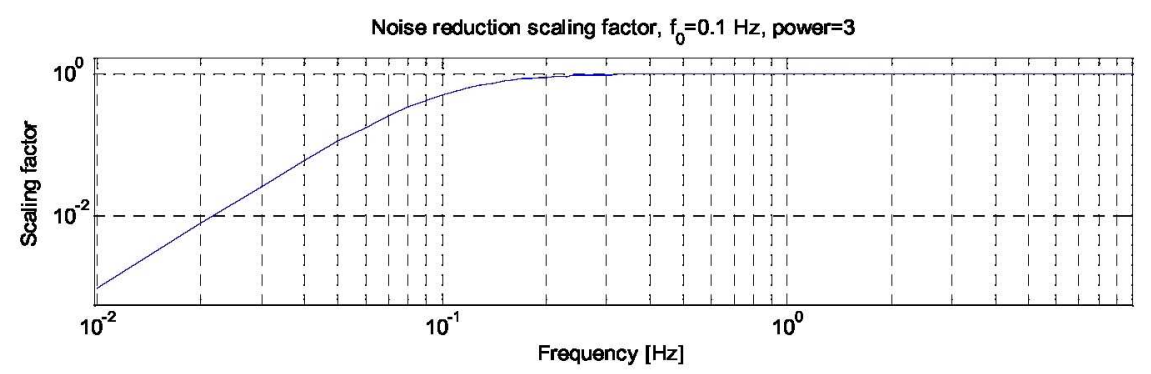

\section{Figure 6. Tukey Noise Reduction Factor.}

The noise floor was judged to not be as great of a concern for the accelerometers placed at two points on the vehicle during rollout. As seen in Fig. 7, the apparent noise floor of the signal seen at these two locations exceeds the projected noise floor of the accelerometers in the frequency range near the first two bending modes. The example plot shown in Fig. 7 is illustrative of trends seen in the other cardinal directions at both of the accelerometer locations. Because these accelerometer signals seemed clean in the ranges important for modal analysis of the system, the signals were filtered using a simple high-pass filter.

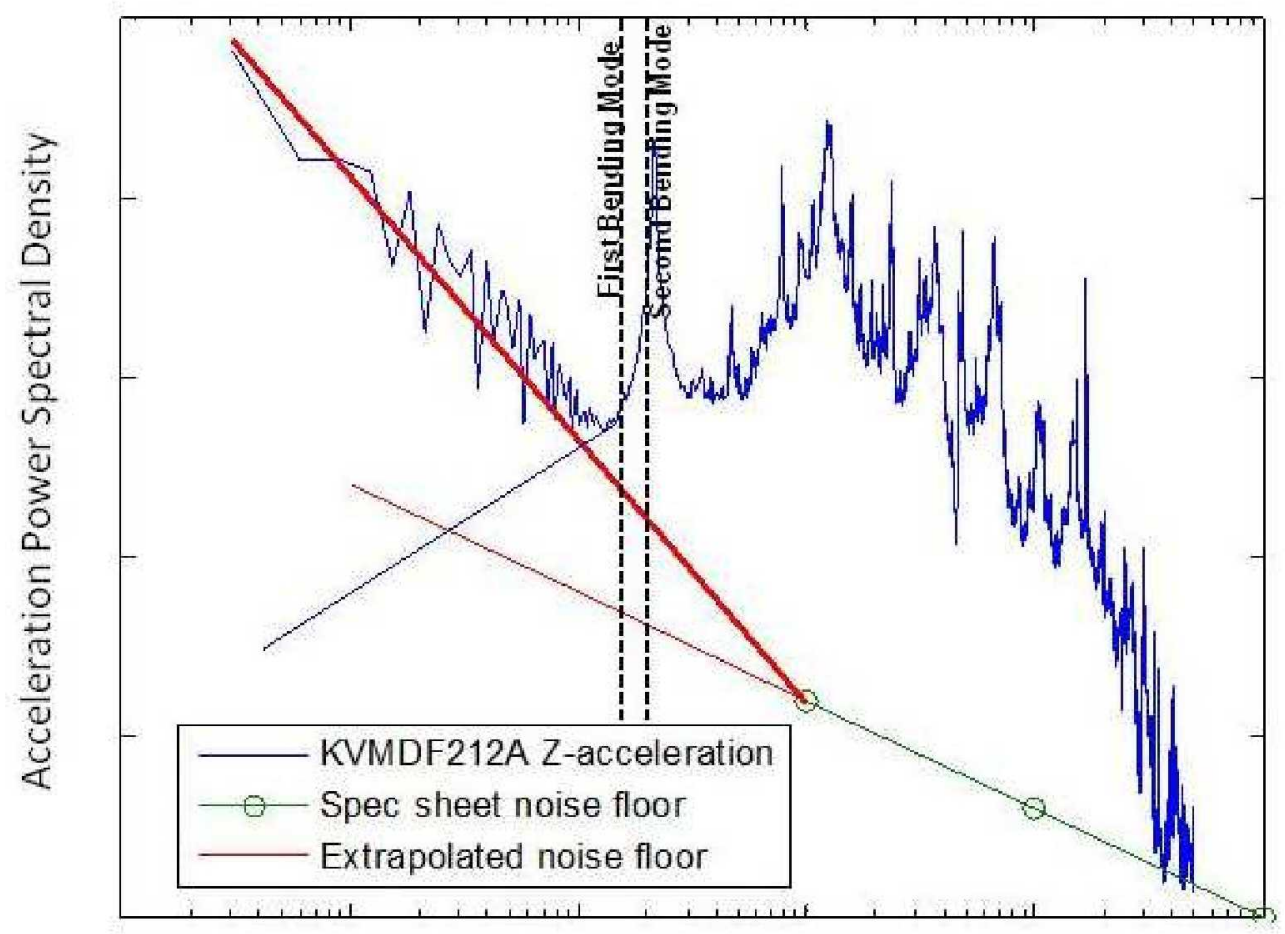

\section{Frequency}

Figure 7. Measured Z-Direction Acceleration PSD at Station A.

\section{B. Base Drive Simulation}

Because of the time period selected for reconstruction the only source of excitation of the combined MLP/Ares I$\mathrm{X}$ finite element model are the base drive accelerations applied at the MLP/CT connection points. In order to simulate the effects of the base drive, the measured and filtered accelerations must be integrated twice to create time histories for the displacements and velocities of the connection points. 
The next step in the analysis procedure is to identify the degrees of freedom associated with the MLP/CT interface points and separate them in the mass and stiffness matrices. For instance, the stiffness matrix can be separated as follows:

$$
[K] \rightarrow\left[\begin{array}{ll}
K_{11} & K_{12} \\
K_{21} & K_{22}
\end{array}\right]
$$

Where $K_{22}$ are the DOF's of the crawler attach points where the base drive acceleration is being applied and $K_{1 I}$ are the remaining un-driven DOF's in the model. This leads to the equation of motion for rollout:

$$
\left[\begin{array}{ll}
M_{11} & M_{12} \\
M_{21} & M_{22}
\end{array}\right]\left[\begin{array}{l}
\ddot{U}_{1} \\
\ddot{U}_{2}
\end{array}\right]+\left[\begin{array}{ll}
C_{11} & C_{12} \\
C_{21} & C_{22}
\end{array}\right]\left[\begin{array}{l}
\dot{U}_{1} \\
\dot{U}_{2}
\end{array}\right]+\left[\begin{array}{ll}
K_{11} & K_{12} \\
K_{21} & K_{22}
\end{array}\right]\left[\begin{array}{l}
U_{1} \\
U_{2}
\end{array}\right]=\left[\begin{array}{l}
0 \\
f
\end{array}\right]
$$

Where $\ddot{U}_{2}$ is the known/measured acceleration that is driving the ML/CLV at the crawler attach points. Likewise, $\dot{U}_{2}$ and $U_{2}$ are the velocity and displacement at the attach points as calculated from the integration of the acceleration. $\mathrm{C}_{11}$ is the modal damping matrix, which is discussed in greater detail at the end of this subsection. Since measured accelerations are applied at the crawler attach points, there is no damping at these points rendering $\mathrm{C}_{22}, \mathrm{C}_{21}$, and $\mathrm{C}_{12}$ essentially zero.

In previous Ares loads cycles, the equations of motion for the system were solved using an enforced displacement technique based on the known behavior at the MLP/CT interfaces and a conversion of the equations of motion into modal coordinates. To simplify the solution process, a Craig-Bampton transformation was used in the Ares I-X reconstruction effort. This transformation is used to decouple the known drive points from the rest of the degrees of freedom in the stiffness matrix. The Craig-Bampton transformation is given by,

$$
\left\{\begin{array}{l}
U_{1} \\
U_{2}
\end{array}\right\}=\Psi_{C B}\left\{\begin{array}{l}
\eta_{1} \\
U_{2}
\end{array}\right\}
$$

where the Craig-Bampton transformation matrix $\left(\Psi_{\mathrm{CB}}\right)$ is given by,

$$
\Psi_{C B}=\left[\begin{array}{cc}
\Phi & \Psi \\
0 & \mathrm{I}
\end{array}\right]
$$

where $\Phi$ are the fixed boundary modes of the system and $\Psi$ are the constraint modes of the system. In addition, at this point the constraint modes are limited to those under $30 \mathrm{~Hz}$. All of the physical velocities and accelerations can be converted using the Craig-Bampton transformation matrix in the same manner shown in Equation 7.

Rearranging the equations of motion using the Craig-Bampton transformation yields the following:

$$
\begin{aligned}
& {\left[\begin{array}{cc}
\mathrm{I} & \Phi^{\mathrm{T}}\left(M_{11} \Psi+M_{12}\right) \\
\left(\Psi^{\mathrm{T}} M_{11}+M_{21}\right) \Phi & \left(\Psi^{\mathrm{T}} M_{11} \Psi+M_{12} \Psi+\Psi^{\mathrm{T}} M_{12}+M_{22}\right)
\end{array}\right]\left\{\begin{array}{c}
\ddot{\eta} \\
\ddot{u}_{2}
\end{array}\right\}+\left[\begin{array}{cc}
\Omega^{2} & 0 \\
0 & \mathrm{I}
\end{array}\right]\left\{\begin{array}{c}
\eta \\
u_{2}
\end{array}\right\}=\left\{\begin{array}{l}
0 \\
f
\end{array}\right\}} \\
& \Downarrow \\
& \ddot{\eta}+\Omega^{2} \eta=-\Phi^{\mathrm{T}}\left(M_{11} \Psi+M_{12}\right) \ddot{\mathrm{u}}_{2}
\end{aligned}
$$

Thus the generalized responses can be found using the measured base drive accelerations alone. However, the accelerations must be integrated to determine the loads due to the constraint modes. Damping is not shown in Equations 8 or 9 but was included in the simulation used for reconstruction of Ares I-X data. The entire system was assumed to be critically damped. In previous Ares I load cycles, the critical damping has been assumed to be twice what was used here but the lower value yielded more accurate reconstructed values for Ares I-X. The equations of motion were integrated using a 4th order Runge Kutta equation to find the modal displacements and velocities. Physical displacements, velocities, and accelerations were calculated based on their modal counterparts.

\section{Overturning Moment Compensation}

An overturning moment is created on a long, slender vehicle like Ares I-X when the force of gravity is not collinear with the vehicle's longitudinal axis. This effect occurs when the base drive acceleration loadings cause large lateral bending deflections of the structure, pushing the center of mass out of line and creating a resulting moment. An adjustment to the system stiffness matrix to account for this effect was included in all of the response computations.

\section{Gravity Effects During Crawler Pickup}

The acceleration due to gravity is not included in the main rollout base drive reconstruction for Ares I-X on account of the fact that the measured accelerometer data has been adjusted to remove it. A separate study of the 
loading due to gravity during the process of picking up the MLP and Ares I-X up off of the columns in the Vehicle Assembly Building (VAB) was carried out. Gravity loads are first found with the MLP/Ares I-X model grounded at the six pad/VAB support columns. The displacements at the column degrees of freedom are then cancelled using forces applied in the direction opposite of gravity. Finally, gravity is applied to the combined model when supported at the four MLP/CT attach points. The total displacements are added up to yield loads due to gravity. One of the interesting findings of this study of the changes in boundary conditions during pickup is that because of the spacing between the various support points, the MLP deflects under the weight of Ares I-X in one direction while supported in the VAB, then in a different direction when supported by the crawler as shown in Fig. 8.

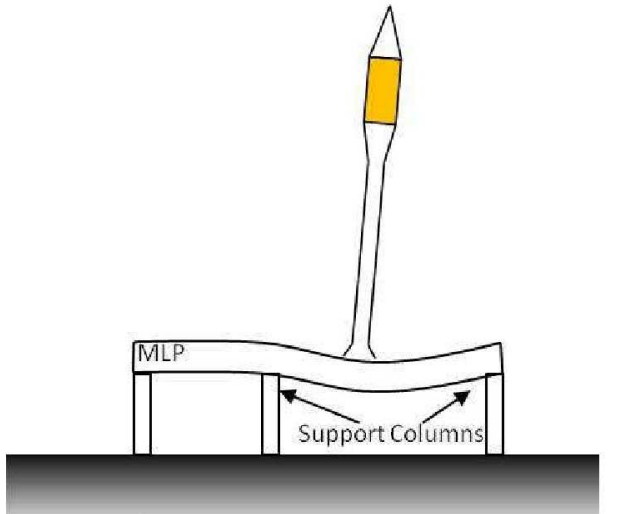

a.) VAB Boundary Conditions



b.) Crawler Boundary Conditions

\section{Figure 8. Exaggerated Changes in Boundary Conditions During Crawler Pickup.}

In a normal analysis cycle for Ares I, wind loads on the vehicle and ML due to ground wind gust, vortex shedding, and locked-in wind-induced oscillation are modeled. As mentioned earlier, separate analysis not discussed here found that the peak winds present during the Ares I-X rollout did produce discernable but very small loads on the vehicle. For this reason, the wind loads have not been included in this analysis.

\section{Results}

The Ares I-X rollout process was simulated for the 6,500 second time period described in Section III-A using the analysis procedures described in III-B. The key metrics for judging the validity of the simulated results come from the measured values of HDP loads as well as accelerations, velocities, and displacements at two points on the vehicle where accelerometers were located. As summarized in Table 1, the simulation results agree quite closely with measured data, particularly in the RMS values when the tapered filtering is used on the base drive acceleration inputs. The displacements at the higher accelerometer (Station A) are particularly accurate.

Table 1. Comparison of Simulation and Measured Results.

\begin{tabular}{|c|c|c|c|c|}
\cline { 2 - 5 } \multicolumn{1}{c|}{} & \multicolumn{2}{c|}{ Simulation } & \multicolumn{2}{c|}{ Measured } \\
\cline { 2 - 5 } \multicolumn{1}{c|}{} & Peak & RMS & Peak & RMS \\
\hline HDP-5 Loads & 0.80 & 0.14 & 1.00 & 0.15 \\
\hline HDP-6 Loads & 1.12 & 0.17 & 1.00 & 0.18 \\
\hline HDP-7 Loads & 1.11 & 0.19 & 1.00 & 0.20 \\
\hline HDP-8 Loads & 0.85 & 0.15 & 1.00 & 0.16 \\
\hline $\mathrm{U}_{\mathrm{y}}$ (Station A) & 1.02 & 0.22 & 1.00 & 0.24 \\
\hline $\mathrm{U}_{\mathrm{z}}$ (Station A) & 0.90 & 0.21 & 1.00 & 0.21 \\
\hline $\mathrm{A}_{\mathrm{y}}$ (Station A) & 0.91 & 0.12 & 1.00 & 0.12 \\
\hline $\mathrm{A}_{\mathrm{z}}$ (Station A) & 0.96 & 0.13 & 1.00 & 0.12 \\
\hline
\end{tabular}

Note: To protect sensitive information, the quantities in the table are all normalized based on the measured peak values but their relative differences from one another remain. 
As seen in Fig. 9, the simulation of the Ares I-X rollout does not capture the full details of the time history of the axial HDP loads. The transducer noise that masks low frequency signals may be responsible for some of the differences between simulated and measured values. It should be noted that the operational Ares I vehicle should less sensitive to low frequency excitation when stabilized through attachment to the new ML tower. Some of the larger features of the axial HDP loads can be seen and the magnitude of the peak response and RMS level are on par with what was measured. In the measured results, there are "blooms" of loading around changes in CT speed. These can be seen in slightly lest distinct spikes in the simulated results.
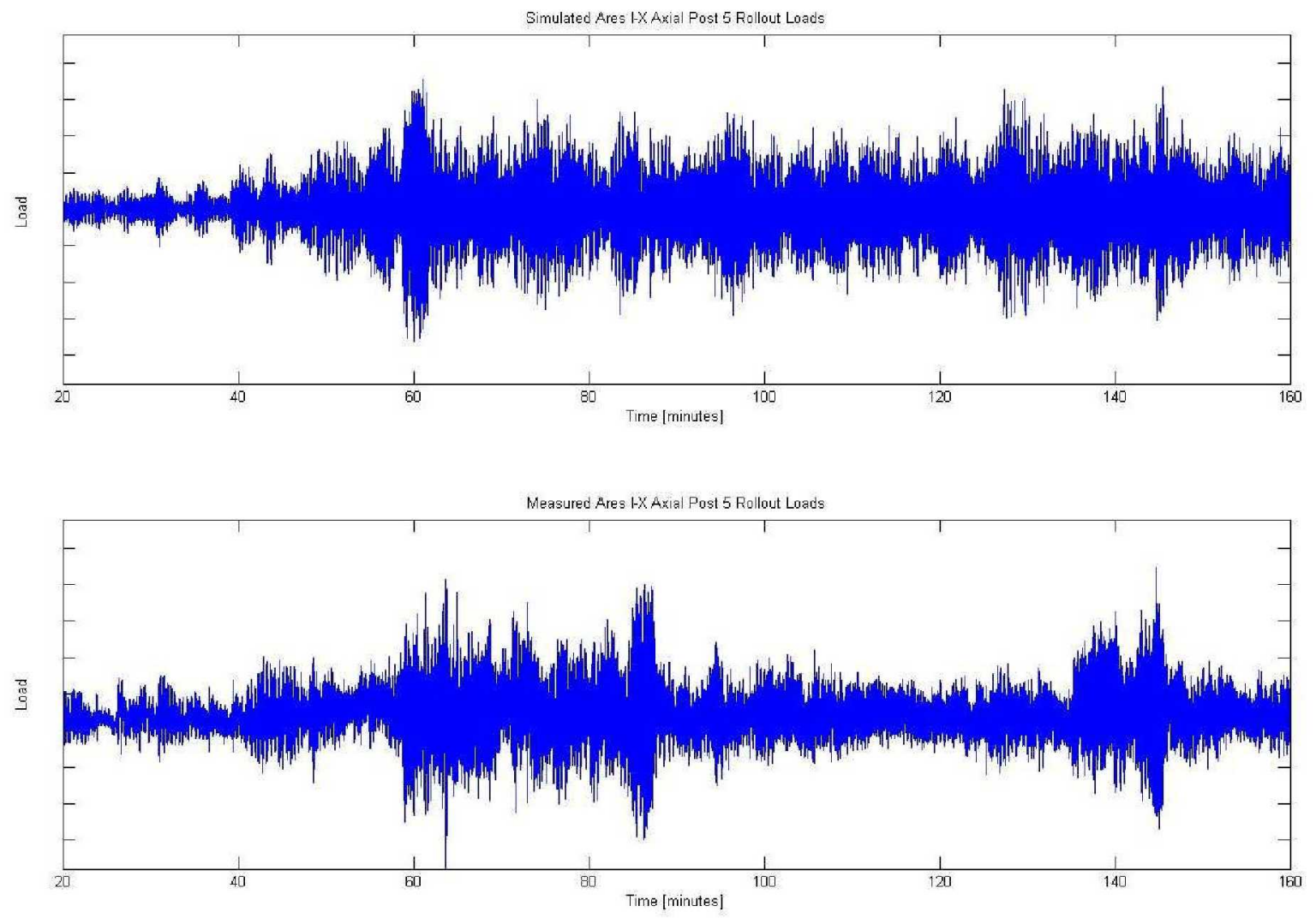

Figure 9. Hold-Down Post 5 Axial HDP Load Time Histories.

The power spectral density for the axial hold-down post loads at Post 5 are plotted in Fig. 10 and show that the simulated results generally closely capture the statistics of the measured values. The primary bending frequencies found using the simulation are a bit lower than their measured values but their magnitudes are similar. The effect of high-pass tapered filtering on the simulated responses is clearly seen in the lower PSD values at low frequencies. 


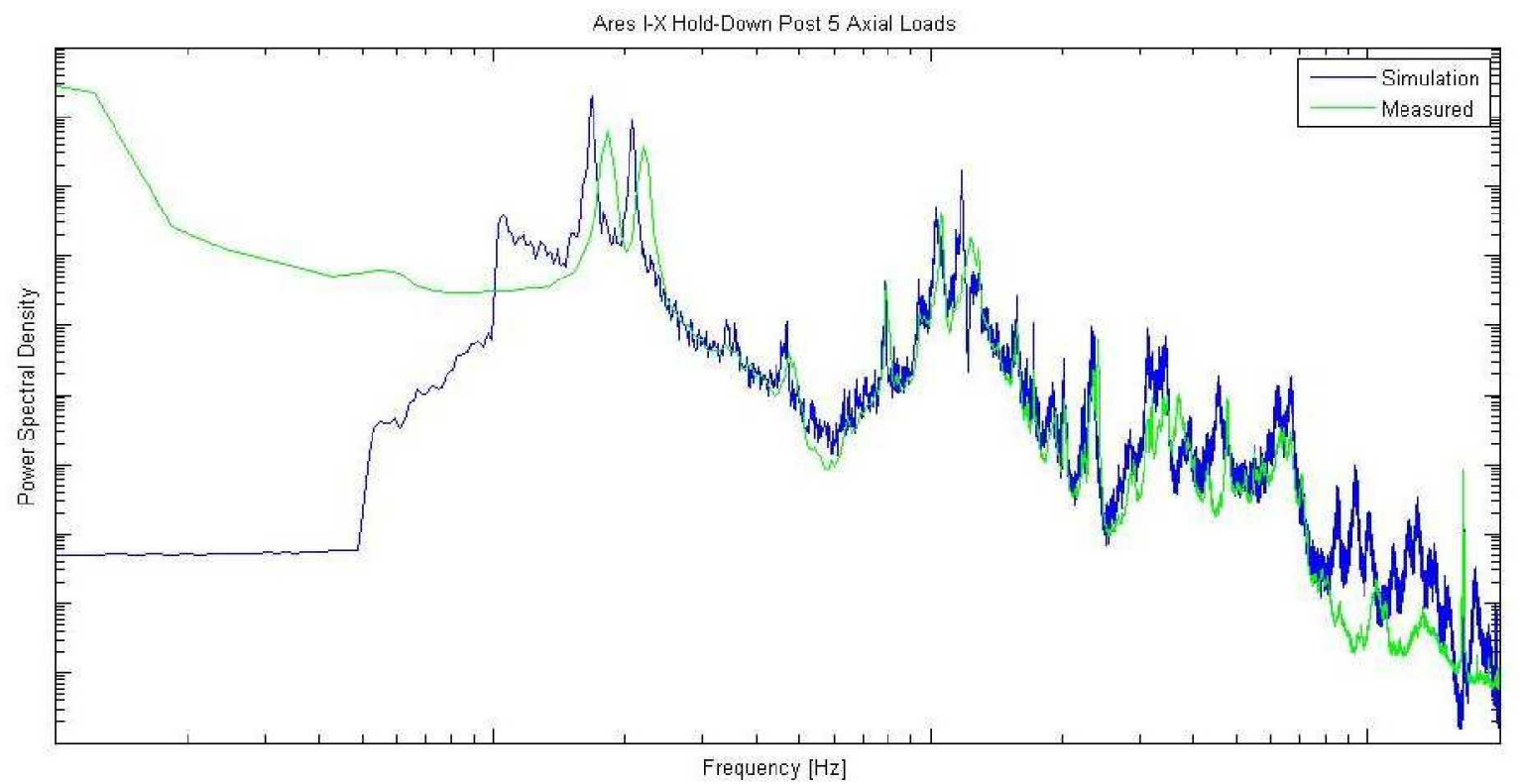

Figure 10. Hold-Down Post 5 Axial HDP Load Time PSDs.

The simulated accelerations in the Z-direction at the higher of the two accelerometers are qualitatively very similar to those that were measured, as seen in Fig. 11. The two responses are similar both in RMS and peak values. The PSD plot in Fig. 12 shows that the frequency content of these two responses is also similar at levels below $7 \mathrm{~Hz}$. Just as with the HDP axial loads, the peaks in the PSD plot are lower for simulated values than for measured values and the effects of the high-pass filtering can again be clearly seen in the simulated results. 

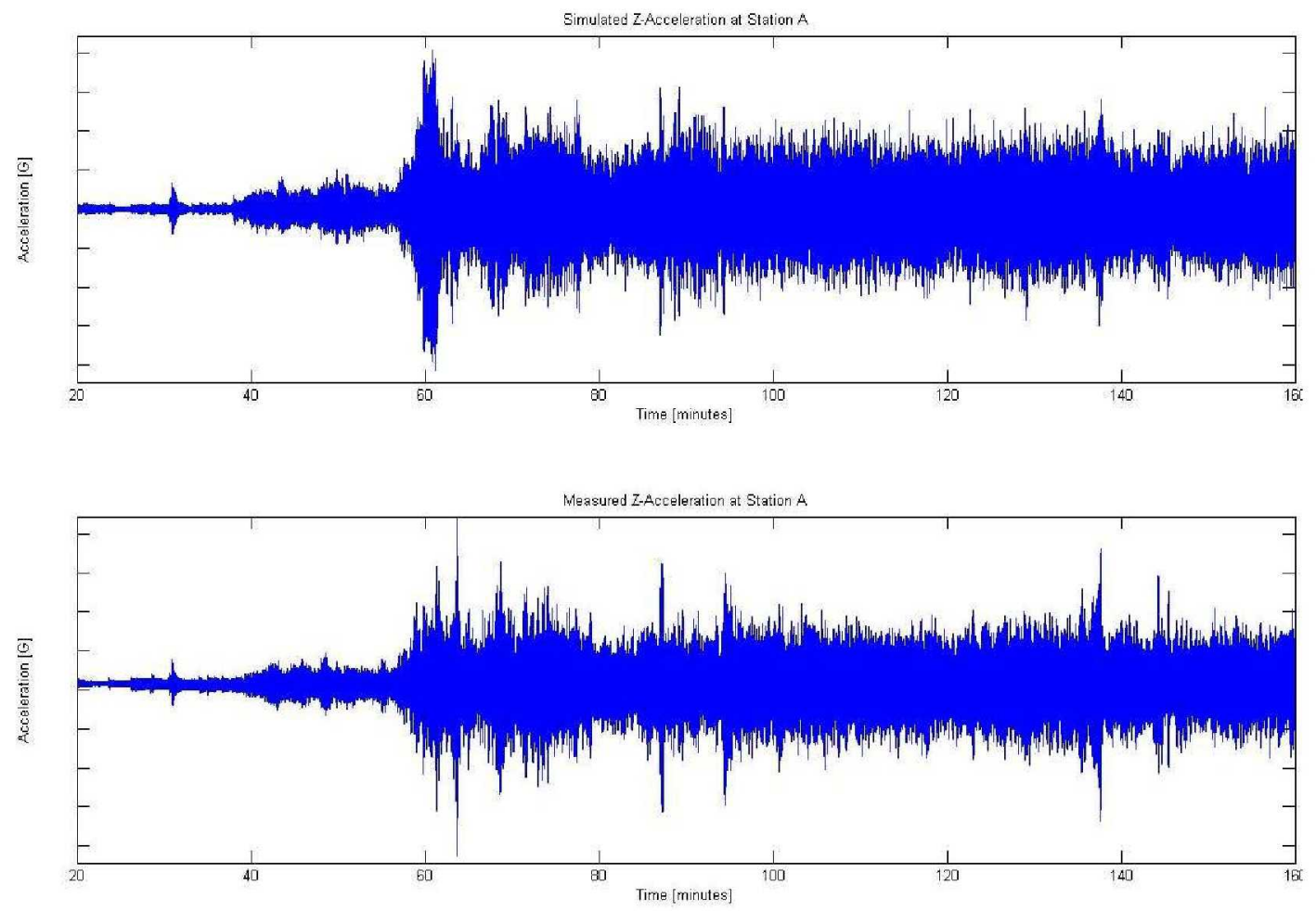

Figure 11. Z-Acceleration Time Histories at Station A.

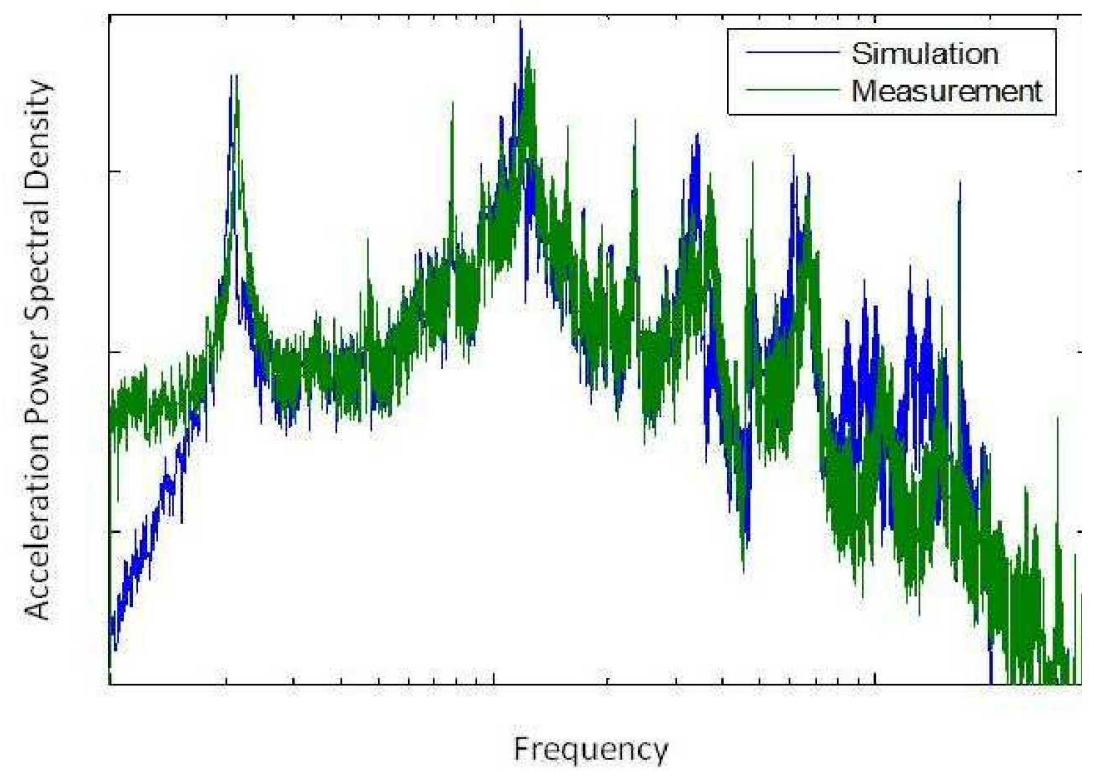

Figure 12. Z-Acceleration PSDs at Station A.

The displacements at Station A show less qualitative similarities the accelerations but peak and RMS values are in good agreement as seen in Fig 13. Some of the differences between the measured and simulated displacements may be the result of the poor characterization of the low frequency drive accelerations. The displacement PSD plot 
shown in Fig. 14 demonstrates that the simulation captures the magnitude of the primary bending mode well but reports a slightly lower frequency once again. The effects of the high-pass filtering are again show clearly.
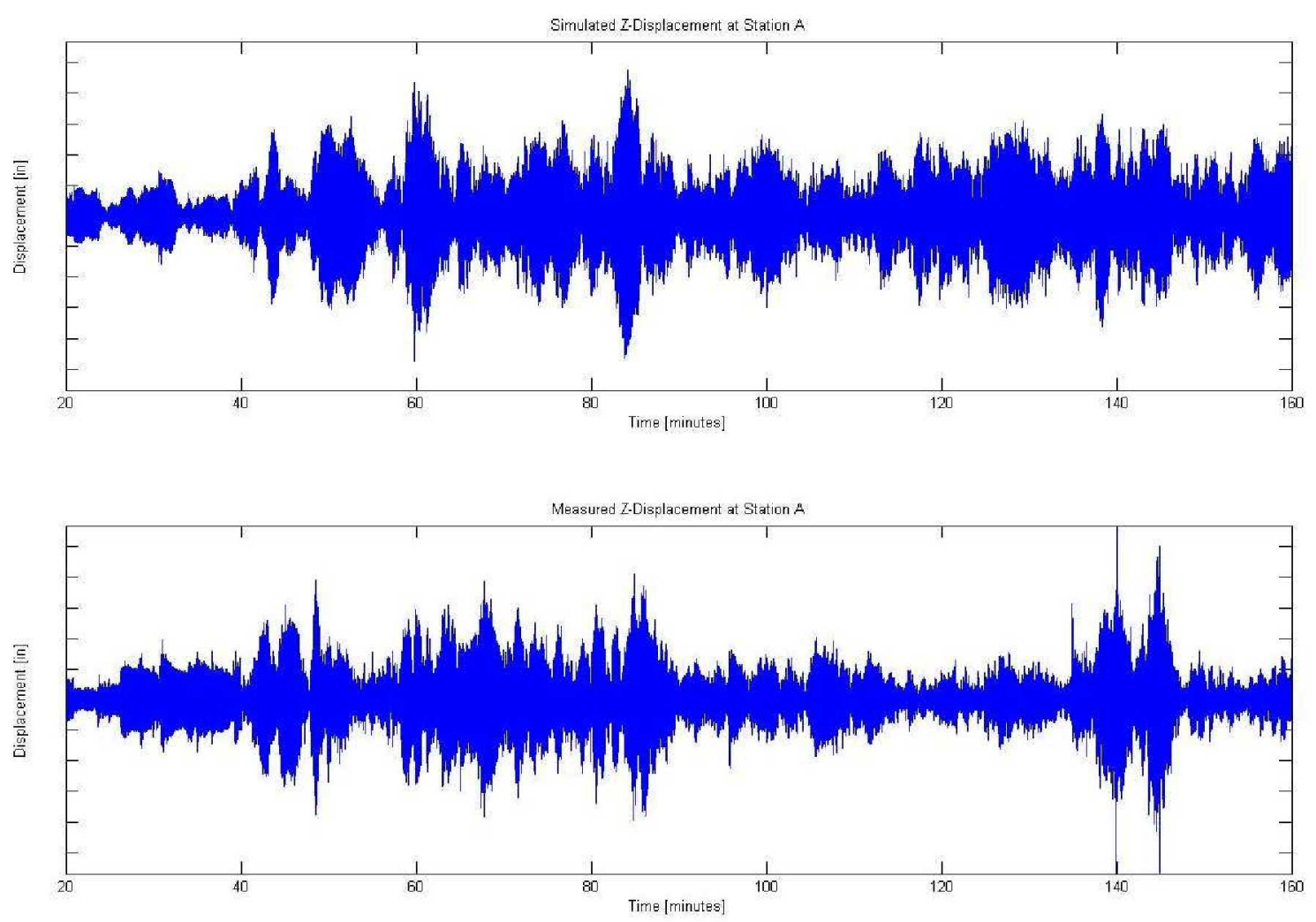

Figure 13. Z-Direction Accelerations Time Histories at Station A. 


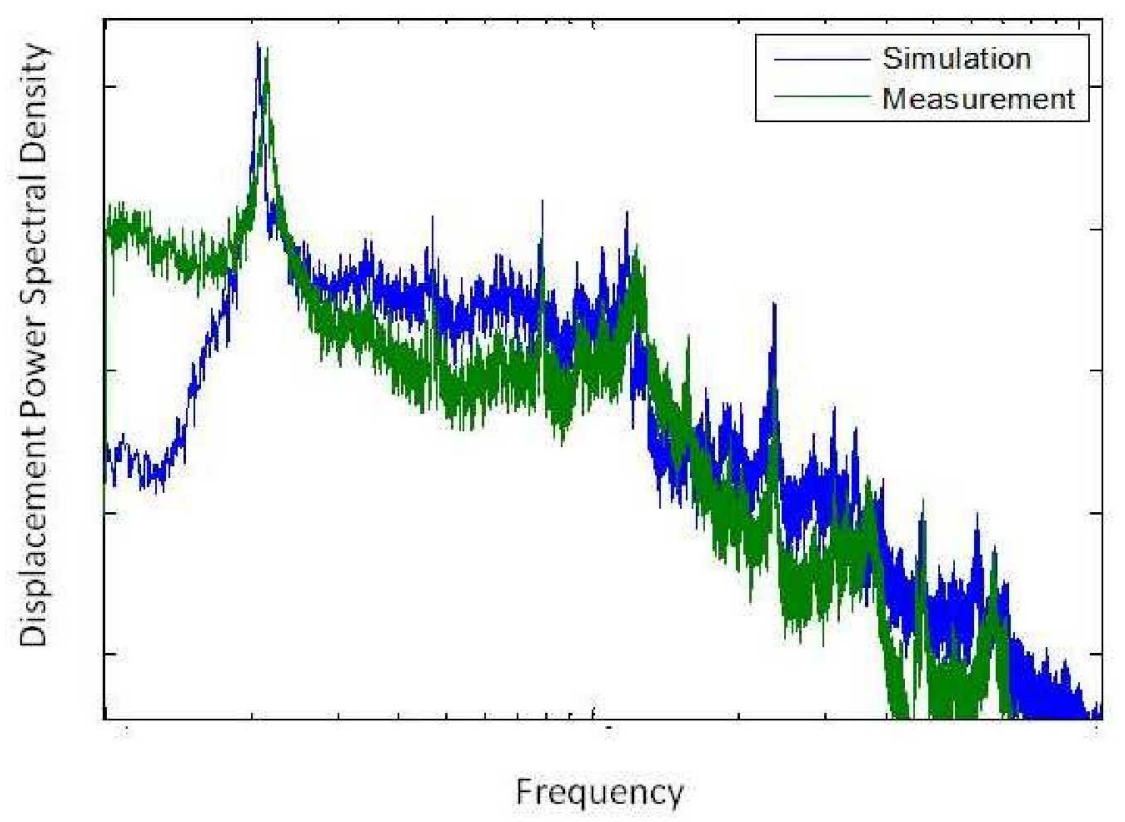

Figure 14. Z-Direction Displacement PSDs at Station A.

\section{Discussion}

It has been shown in this study that the Ares I simulation tools do conservatively estimate rollout loads when used to model Ares I-X. While not every detail of the time histories can be exactly produced, the trends and magnitudes of the real loads seen while rolling out Ares I-X were accurately reconstructed with some margin of safety. Hold-down post peak simulated values were roughly $20 \%$ higher than those measured while RMS values were roughly $40 \%$ higher. Simulated acceleration values at Station A high up on the vehicle had both peak and RMS values only $5 \%$ higher than measured.

It is noted that the Ares I-X vehicle was different from the planned Ares I vehicle layout. It lacked a powered upper stage, Orion crew capsule, launch abort system (LAS), and the vibration damping systems meant to deal with thrust oscillation during flight. The first stage solid rocket booster was made up of four segments with a dummy upper fifth segment whereas the Ares I would have five segments filled with propellant. In addition to the differences in the vehicle itself, the support equipment used during rollout differs from that which will be used with Ares I. The Ares I-X rollout made use of heritage equipment including a Mobile Launcher Platform (MLP) used in the Space Shuttle program. While the structure of the crawler, MLP, and Ares I-X differs from the planned operational structure for Ares I during rollout, it is felt that the lessons learned from reconstructing the loads on a vehicle similar to Ares I far outweigh the uncertainties due to its dissimilarities.

It is also noted that the base drive accelerations used to drive the finite element models of Ares I-X on the MLP were produced by accelerometers whose noise floors were too high to accurately capture important low-frequency signals. These noise floors exceeded the first two bending modes of the combined Ares I-X/MLP model, meaning that signals in that low range are tainted and likely lead to higher loads. This situation is particularly troublesome for Ares I-X with its low-frequency modes, but it will be of less concern for Ares I as the vehicle will ride on a new ML with a tower and will be stabilized at two points. With the stabilization provided by the tower and two arms, the Ares I bending modes will be above the noise floor of the accelerometers.

The results of this study have yielded increased confidence in the ability to use Ares I simulation and modeling tools to accurately but conservatively model vehicle loads during rollout. The results have also yielded slight changes in both the modeling of critical damping and in the methods for simulating the base drive accelerations. The filtered data gathered during the Ares I-X rollout will continue to be used in simulation of Ares I rollout loads in future loads cycles. 